\title{
Tetra- $n$-butyl ammonium bromide semi-clathrate hydrate process for post-combustion capture of carbon dioxide in the presence of dodecyl trimethyl ammonium chloride
}

\author{
Xiao-Sen $\mathrm{Li}^{\mathrm{a}, \mathrm{b}, *}$, Chun-Gang Xu ${ }^{\mathrm{a}, \mathrm{b}, \mathrm{c}}$, Zhao-Yang Chen ${ }^{\mathrm{a}, \mathrm{b}}$, Hui-Jie $\mathrm{Wu}^{\mathrm{a}, \mathrm{b}}$ \\ ${ }^{a}$ Key Laboratory of Renewable Energy and Gas Hydrate, Guangzhou Institute of Energy Conversion, Chinese Academy of Sciences, Guangzhou 510640, PR China \\ ${ }^{\mathrm{b}}$ Guangzhou Center for Gas Hydrate Research, Chinese Academy of Sciences, Guangzhou 510640, PR China \\ ${ }^{\mathrm{c}}$ Graduate University of Chinese Academy of Sciences, Beijing 100083, PR China
}

\section{A R T I C L E I N F O}

\section{Article history:}

Received 5 February 2010

Received in revised form

3 June 2010

Accepted 5 June 2010

\section{Keywords:}

$\mathrm{CO}_{2}$ hydrate

Flue gas

TBAB

DTAC

Semi-clathrate hydrate

\begin{abstract}
A B S T R A C T
To determine the appropriate operating conditions for separating carbon dioxide from flue gas via the hydrate formation, the effects of the concentrations of dodecyl trimethyl ammonium chloride (DTAC) in $0.29 \mathrm{~mol} \%$ Tetra- $n$-butyl ammonium bromide (TBAB) aqueous solution and the initial pressures on the induction time of the hydrate formation and $\mathrm{CO}_{2}$ separation efficiency are investigated. The experiments are conducted at the DTAC concentration range of $0-0.056 \mathrm{~mol} \%$, initial pressures range of $0.66 \mathrm{MPa}-2.66 \mathrm{MPa}$ and temperature range of $274.95 \mathrm{~K}-277.15 \mathrm{~K}$. The results indicate that the initial pressure of $1.66 \mathrm{MPa}$ in conjunction with the concentration of $0.028 \mathrm{~mol} \% \mathrm{DTAC}$ is most favorable for $\mathrm{CO}_{2}$ separation. At the condition, the induction time of forming the hydrate can be shortened considerably and $\mathrm{CO}_{2}$ can be purified from $17.0 \mathrm{~mol} \%$ to $99.4 \%$ with the two-stage hydrate separation process. $\mathrm{CO}_{2}$ split fractions for Stage 1 and Stage 2 are 0.54 and 0.39, respectively, and the separation factors are 9.60 and 62.25 , respectively.
\end{abstract}

Crown Copyright $\odot 2010$ Published by Elsevier Ltd. All rights reserved.

\section{Introduction}

Carbon dioxide $\left(\mathrm{CO}_{2}\right)$ as one main greenhouse gas contributes itself to greenhouse effect accounted for about $60 \%$ [1]. In order to deal with the challenge of global warming and carry out the Kyoto protocol, it is imperative to reduce the emission of $\mathrm{CO}_{2}$, especially for the $\mathrm{CO}_{2}$ emitted from fossil fuel power plants. Various methods such as cryogenic fractionation, selective adsorption, gas absorption and membrane process, have been proposed. However, the above methods have their individual issues of either high corrosion, large energy consumption, high cost, or low capacity [2]. Accordingly, a new efficient and more cost-effective technology which is different from the conventional methods needs to be explored.

The hydrate separation method for gas mixtures is a novel gas separation technique. Since Glew [3] applied the first patent and successfully separated propane and propylene from gas mixture by forming the hydrate with structure II (sII), the method for gas

\footnotetext{
* Corresponding author. Key Laboratory of Renewable Energy and Gas Hydrate, Guangzhou Institute of Energy Conversion, Chinese Academy of Sciences, Guangzhou 510640, PR China. Tel./fax: +86 2087057037.

E-mail address: lixs@ms.giec.ac.cn (X.-S. Li).
}

separation has been proposed [3,4]. Recently, separating $\mathrm{CO}_{2}$ from Integrated Gasification Combined Cycle (IGCC) syngas or flue gas via the hydrate formation has attracted many attentions. Preliminary economic assessment shows that the cost of the hydrate technique for $\mathrm{CO}_{2}$ separation from IGCC power plant is approximately 10 U.S. dollars/ton of $\mathrm{CO}_{2}$, which is less than half of that of others [5].

The basis for the separation is the selective partition of the target component between the hydrate phase and the gaseous phase. Because the equilibrium hydrate formation pressure of $\mathrm{CO}_{2}$ is much lower than that of $\mathrm{N}_{2}$ at the same temperature, it is expected that $\mathrm{CO}_{2}$ preferentially encaged into the hydrate crystal phase. The hydrate crystals are separated and subsequently decomposed to create the $\mathrm{CO}_{2}$-rich stream, while the rest constitute the $\mathrm{CO}_{2}$-lean one. For $\mathrm{CO}_{2}$ hydrate separation from flue gas, the first task is to decrease the operating pressure to save the separation cost. The equilibrium hydrate formation pressure of flue gas is between those of pure $\mathrm{CO}_{2}$ and pure $\mathrm{N}_{2}$, and it increases with the decrease of the $\mathrm{CO}_{2}$ concentration. Due to the fact that flue gas from the power plant usually consists of $\mathrm{CO}_{2}$ from 15 to $20 \mathrm{~mol} \%$ [6], the equilibrium pressure for this kind of gas is relatively high. For example, the equilibrium pressures for gas containing $\mathrm{CO}_{2}$ of $17.61 \%$ are $7.60 \mathrm{MPa}$ and $11.00 \mathrm{MPa}$ at $274.00 \mathrm{~K}$ and $277.00 \mathrm{~K}$, respectively [7]. The main challenge of hydrate technique for $\mathrm{CO}_{2}$ separation is 
that it needs the high equilibrium hydrate formation pressure. Hence, a suitable promoter is essential to help in reducing the hydrate formation pressure and the energy consumption without impacting the separation efficiency or $\mathrm{CO}_{2}$ recovery.

Tetrahydrofuran (THF) was chosen as a hydrate promoter by Kang and Lee [8] studying on the thermodynamic verification of recovery of $\mathrm{CO}_{2}$ from flue gas using gas hydrate through phase equilibrium measurements. The result showed that, in principle, $\mathrm{CO}_{2}$ can be purified to $99 \%$ from flue gas containing $17 \% \mathrm{CO}_{2}$ in the presence of THF through a three-stage separation process. Meanwhile, it showed that THF, as an effective additive, can greatly reduce hydrate formation pressure at the temperature range of $273-283 \mathrm{~K}$. However, the induction time was not shortened remarkably. THF was also used by Linga et al., [6] for $\mathrm{CO}_{2}$ capture from flue gas containing $16.9 \% \mathrm{CO}_{2}$ via a medium-pressure clathrate hydrate process. It showed that THF $(1.0 \mathrm{~mol} \%)$ could substantially decrease the pressure from $7.8 \mathrm{MPa}$ to $2.5 \mathrm{MPa}$ at $273.75 \mathrm{~K}$. In addition, THF was found by Kumar et al. [9] to have the function on shortening the induction time and accelerating the hydrate growth compared to the pure water system. Ding [10] used sodium dodecyl sulfate (SDS) associated with an anionic fluorosurfactant (FS-62) (FS-62/SDS: $100 / 1000 \mathrm{ppm}$ ) as a joint additive to capture and sequester $\mathrm{CO}_{2}$ from $\mathrm{CO}_{2} / \mathrm{N}_{2}$ mixture. However, its effect on raising the storage of $\mathrm{CO}_{2}$ in hydrate was limited.

As an environmental friendly compound, Tetra- $n$-butyl ammonium bromide (TBAB) is also an excellent hydrate promoter and it can form semi-hydrate with water molecules and small gas molecules and moderate the hydrate formation condition significantly [11]. The properties of this semi-hydrate such as temperature and enthalpy of dissociation have already been studied [12-14]. TBAB semi-clathrate hydrates technology is also applied for $\mathrm{CO}_{2}$ separation from flue gas. Ahmadloo et al. [15] have done a research on gas separation and storage by forming $\mathrm{CO}_{2}$-TBAB semi-clathrate hydrates at a moderate pressure. His work verified that the concentration of TBAB in the aqueous solution positively impacted the separation of $\mathrm{CO}_{2}$. However, when the concentration of TBAB is higher than 35 mass\%, the recovery of gas has no considerable change. Phase behavior of TBAB semi-clathrate hydrate crystal with several gas components was investigated by Oyama et al. [16]. They found that TBAB was not a gas hydrate formation promoter; but it can promote the hydrate stability. However, Nguyen Hong Duc et al. [2] used TBAB as a hydrate formation promoter to separate $\mathrm{CO}_{2}$ from $\mathrm{CO}_{2} / \mathrm{N}_{2}$ mixture gas by forming gas hydrate. In their work, the hydrate formation conditions and gas storage capacity of the hydrate for a wide range of additive concentrations were determined. Meanwhile, based on the thermodynamic results, they made a commercial flow-sheeting simulator to estimate the operative cost of a continuous $\mathrm{CO}_{2}$ capture by hydrate system. However, the further studies required to carry out efficiently shorten the induction time of the hydrate formation and the gas storage capacity of the hydrate based on the function of TBAB to enhance $\mathrm{CO}_{2}$ recovery efficiency from the flue gas.

In this work, a novel hydrate promoter is used for enhancing the $\mathrm{CO}_{2}$ separation from the $\mathrm{CO}_{2} / \mathrm{N}_{2}$ mixture gas, based on our previous study [17]. The experiments are conducted to separate $\mathrm{CO}_{2}$ from the $\mathrm{CO}_{2} / \mathrm{N}_{2}$ gas mixture by forming semi-clathrate hydrates using $0.29 \mathrm{~mol} \%$ TBAB in conjunction with dodecyl trimethyl ammonium chloride (DTAC) with the different concentrations as an additive. DTAC is compatible with cationic or nonionic surfactants and is widely used as an environmental friendly surfactant for textile materials, pictures, paintings, construction materials, carpentry, etc. [18]. The effects of the concentration of DTAC and the initial pressure on the induction time of forming hydrate and $\mathrm{CO}_{2}$ recovery efficiency are investigated. In addition, a two-stage hydrate separation process is proposed to obtain $\mathrm{CO}_{2}$ with high purity.

\section{Experimental}

\subsection{Experimental apparatus and materials}

The experimental apparatus is shown in Fig. 1A crystallizer (CR) is immersed in a temperature-controlled bath. The crystallizer with an inner volume of $56.4 \mathrm{~cm}^{3}$ is made of 316 stainless steel. The crystallizer has two circular viewing windows made of Plexiglass on the front and back. Mixing of the contents in the CR is accomplished using a magnetic stir bar which is magnetically coupled to a set of rotating magnets (Shanghai Hongpu instrument, Ltd., China) placed directly underneath the crystallizer. The temperature of the liquid phase in the $\mathrm{CR}$ is measured using a Pt1000 thermoprobe (JM6081) with an uncertainty of $\pm 0.1 \mathrm{~K}$. All pressure measurements are determined with Setra smart pressure transducers (model 552, Boxborough, MA, USA) with the pressure range of $0-25 \mathrm{MPa}$ with $\pm 0.02 \mathrm{MPa}$ accuracy. The signals of the pressure and temperature are acquired by a data acquisition system. A gas chromatography (HP6890) is connected with the CR to measure the composition of the gas phase in the $\mathrm{CR}$.

$\mathrm{CO}_{2} / \mathrm{N}_{2}$ gas mixture containing $17.0 \mathrm{~mol} \% \mathrm{CO}_{2}$ and $65.0 \mathrm{~mol} \%$ $\mathrm{CO}_{2}$ are used in the work to simulate flue gas mixture, which is supplied by Foshan Huate Gas Co. TBAB and DTAC are supplied by Xiamen Pioneer Chemical Reagent Co. with a purity of $99.9 \%$. The de-ionized water used with the resistivity of $18.25 \mathrm{~m} \Omega \mathrm{cm}^{-1}$ was produced by an ultra-pure water system supplied by Nanjing Ultrapure Water Technology Co.

\subsection{Experimental procedure}

In our previous work [17], it was found that the TBAB concentration has no significant effect on reducing the pressure in the system (the pressure change reflects the gas storage capacity of the hydrate) in the process of hydrate formation when its concentration is more than $0.29 \mathrm{~mol} \%[3,17]$. Hence, in this work, TBAB of $0.29 \mathrm{~mol} \%$ is used as the main promoter for $\mathrm{CO}_{2}$ hydrate formation in the experiment. DTAC $(0,0.014 \%, 0.028 \%$, and $0.056 \%)$ as a secondary promoter is dissolved into the TBAB solution. The water bath temperature $(\mathrm{T})$ is set at $274.95 \mathrm{~K}$ and $277.15 \mathrm{~K}$ respectively.

Prior to the experiment, the $\mathrm{CR}$ was cleaned using the de-ionized water and allowed to dry. Then $0.29 \mathrm{~mol} \% \mathrm{TBAB}$ aqueous solution in conjunction with DTAC prepared at a desired concentration was introduced into the $\mathrm{CR}$ to a desired volume. Subsequently, the $\mathrm{CR}$ with the solution was flushed with $\mathrm{CO}_{2} / \mathrm{N}_{2}$ mixture gas at least four

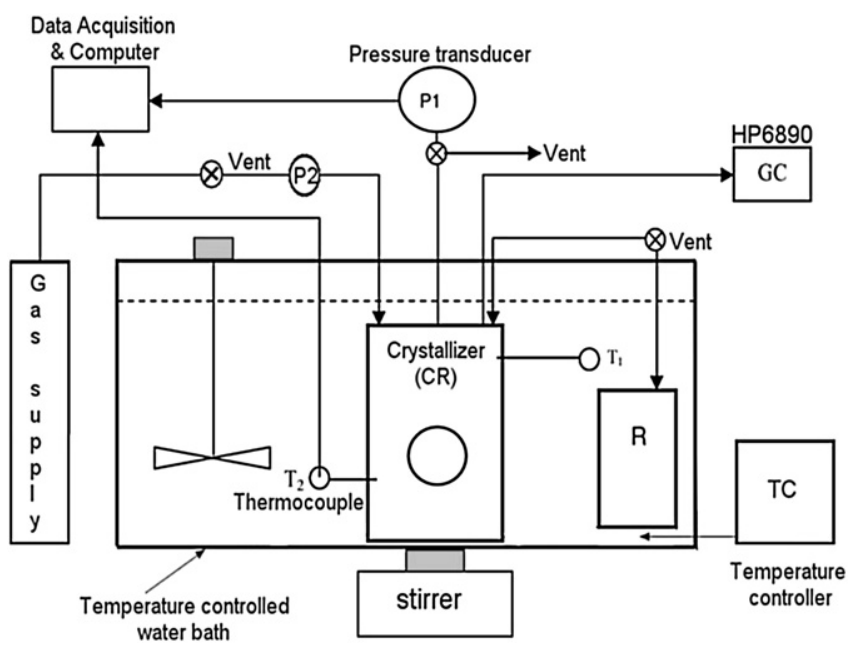

Fig. 1. Schematic of experimental apparatus. 
times to ensure that it was air-free, and then $\mathrm{CO}_{2} / \mathrm{N}_{2}$ mixture gas was charged into the cell until the desired pressure. Once the temperature was stabilized (typically within $2 \mathrm{~min}$ ) the stirrer in the CR was started at the speed of $600 \mathrm{rpm}$ and the experimental time also began to be recorded. During the experiment, the temperature and pressure in the system were recorded. After the system pressure is maintained constant for more than half an hour, the hydrate formation is considered to complete. The stirrer was stopped and the residual gas was sampled and analyzed with the gas chromatography. Then, the vent valve was opened, and the residual gas was quickly purged. Subsequently, the vent was closed, and the vessel was warmed to room temperature to allow the hydrate dissociate completely. The decomposed gas and dissolved gas at the relative high pressure released from the hydrate slurry phase were collected and analyzed by the gas chromatography (GC). The method was adopted elsewhere [15].

\section{3. $\mathrm{CO}_{2}$ recovery and efficiency}

$\mathrm{CO}_{2}$ separation efficiency can be given from the two following equations [9]. The $\mathrm{CO}_{2}$ recovery or split fraction (S. Fr.) of carbon dioxide is expressed as:

S.Fr. $=\frac{n_{\mathrm{CO}_{2}}^{\mathrm{H}}}{n_{\mathrm{CO}_{2}}^{\mathrm{Feed}}}$

The separation factor (S.F.) is expressed as:

$$
\mathrm{S} . \mathrm{F}=\frac{n_{\mathrm{CO}_{2}}^{\mathrm{H}} \times n_{\mathrm{N}_{2}}^{\text {gas }}}{n_{\mathrm{N}_{2}}^{\mathrm{H}} \times n_{\mathrm{CO}_{2}}^{\mathrm{gas}}}
$$

where $n_{\mathrm{CO}_{2}}^{\text {gas }}, n_{\mathrm{CO}_{2}}^{\mathrm{H}}$ and $n_{\mathrm{CO}_{2}}^{\mathrm{Feed}}$ are the moles of $\mathrm{CO}_{2}$ in the gas phase, in the hydrate slurry phase at the end of the hydrate formation, and in the initial gas mixture, respectively. $n_{\mathrm{N}_{2}}^{\text {gas }}, n_{\mathrm{N}_{2}}^{\mathrm{H}}$ are the moles of $\mathrm{N}_{2}$ at the end of the hydrate formation and the moles of $\mathrm{N}_{2}$ in the hydrate slurry phase, respectively.

\section{Results and discussion}

\subsection{Induction time of hydrate formation}

In the experiment, we firstly determined whether $\mathrm{CO}_{2} / \mathrm{N}_{2}$ gas mixture could form gas hydrate in pure DTAC solution at the bath temperature range from $274.95 \mathrm{~K}$ to $277.15 \mathrm{~K}$ and the initial pressure range from $0.66 \mathrm{MPa}$ to $2.66 \mathrm{MPa}$. The result showed that $\mathrm{CO}_{2} /$ $\mathrm{N}_{2}$ mixture gas could not form any gas hydrate in DTAC solution in absence of TBAB under the above condition.

Fig. 2 gives the change of the induction time of the hydrate formation for the $\mathrm{CO}_{2} / \mathrm{N}_{2}$ gas mixture with $17.0 \mathrm{~mol} \% \mathrm{CO}_{2}$ in the $0.29 \mathrm{~mol} \%$ TBAB aqueous system in the presence of DTAC with the different concentrations vs. the initial pressure at $274.95 \mathrm{~K}$. It can be seen from Fig. 2 that the addition of DTAC results in a remarkable reduction of the induction time, and the induction time decreases with the increase of concentration of DTAC at the fixed initial pressure. For example, when the initial pressure is $1.66 \mathrm{MPa}$, the induction time for pure TBAB aqueous solution is $31.0 \mathrm{~min}$. However, when DTAC with the concentration from $0.014 \mathrm{~mol} \%$ to $0.056 \mathrm{~mol} \%$ is added into the 0.29 mol\% TBAB aqueous solution, the induction time is shortened from $7.1 \mathrm{~min}$ to $3.0 \mathrm{~min}$. It may be due to the fact that DTAC as a surfactant can change the surface activity of the solution and lower its surface tension. As a result, the addition of DTAC promotes the dissolving ability of mixture gases in the TBAB solution, and furthermore enhances the degree of super-saturation of $\mathrm{CO}_{2}$. According to the expression for the rate of hydrate nucleation $(J)$ given by Nataraja [19],

$J=k \times(S-1)^{n}$

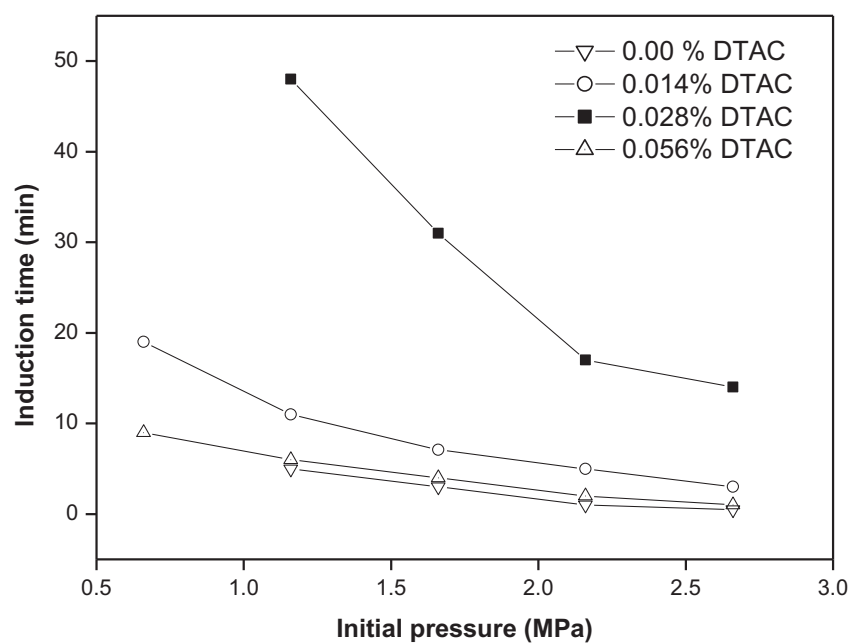

Fig. 2. Induction time of the hydrate formation for $17.0 \mathrm{~mol} \% \mathrm{CO}_{2} / \mathrm{N}_{2}$ gas mixture in TBAB aqueous system in the presence of DTAC with different concentrations vs. initial pressure at $274.95 \mathrm{~K}$.

where $k$ and $n$ are the constants, and $S$ is the super-saturation ratio; it can be seen that the nucleation rate increases with the degree of super-saturation resulting in the decrease of the induction time. However, as shown in Fig. 2, the reduction of the induction time is quite small, when the concentration of DTAC changes from $0.028 \mathrm{~mol} \%$ to $0.056 \mathrm{~mol} \%$ at the fixed initial pressure. According to the characteristics of the surfactant $[20,21]$, the surface tension of solution is the smallest when the concentration of DTAC is critical micellar concentration (CMC) of $1.6 \times 10^{-2} \mathrm{~mol} / \mathrm{L}$ which is estimated by use of the way of Zhong [22]. Its mole percentage is approximately $0.028 \mathrm{~mol} \%$. However, when the concentration exceeds its CMC, the increase of concentration cannot change surface activity any more, and the surface tension cannot be also further decreased. Thus, the super-saturation of $\mathrm{CO}_{2}$ in the solution can also not rise any more. Hence, the induction time of the hydrate formation cannot be shortened in spite of the increase of the DTAC concentration. This is the reason why the induction time has little change with the increase of the DTAC concentration from $0.028 \mathrm{~mol}$ $\%$ to $0.056 \mathrm{~mol} \%$. The similar phenomenon was found by Watanabe et al. [23]. In addition, it can be also seen from Fig. 2 that the induction time reduces with the increase of the initial pressure. This is because the higher initial pressure creates the higher supersaturation which results in the higher nucleation rate.

Likewise, Fig. 3 gives a typical comparison for the induction time of hydrate formation for $65.0 \mathrm{~mol} \% \mathrm{CO}_{2} / \mathrm{N}_{2}$ gas mixture as the feed gas for the second-stage separation with and without the addition of DTAC. It can be seen from Fig. 3 that a small amount of DTAC reduces the induction time significantly at $277.15 \mathrm{~K}$. For example, the induction time with $0.028 \mathrm{~mol} \%$ DTAC is about $0.4 \mathrm{~min}$ and is one thirteenth of that without DTAC at initial pressure of $1.66 \mathrm{MPa}$. In addition, it can be found from Figs. 2 and 3 that for $65.0 \mathrm{~mol} \%$ $\mathrm{CO}_{2} / \mathrm{N}_{2}$ mixture gas as the feed gas for the second-stage separation, the addition of DTAC can remarkably reduce the induction time of the hydrate formation, compared with that for $17.0 \mathrm{~mol} \% \mathrm{CO}_{2} / \mathrm{N}_{2}$ mixture gas. It is because $65.0 \mathrm{~mol} \% \mathrm{CO}_{2} / \mathrm{N}_{2}$ mixture gas has the higher component of $\mathrm{CO}_{2}$. Furthermore, it is easier to be induced to form the hydrate under the function of DTAC.

\subsection{Pressure drop during hydrate formation}

Fig. 4 gives the pressure drops in the system vs. the DTAC concentration in $0.29 \mathrm{~mol} \% \mathrm{TBAB}$ aqueous solution with the different initial pressures at $274.95 \mathrm{~K}$ using $17.0 \mathrm{~mol} \% \mathrm{CO}_{2} / \mathrm{N}_{2}$ 


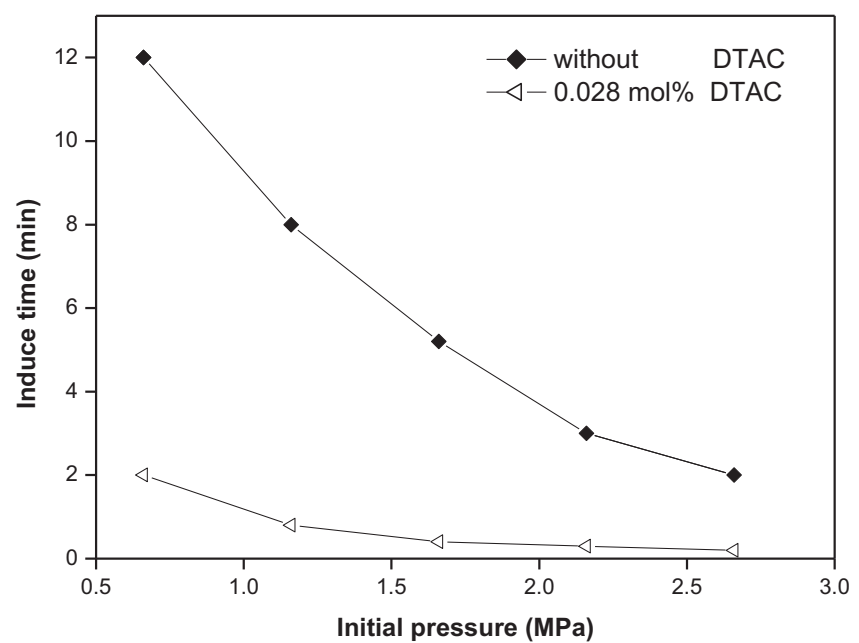

Fig. 3. Induction time of the hydrate formation for $65.0 \mathrm{~mol} \% \mathrm{CO}_{2} / \mathrm{N}_{2}$ mixture gas in $0.29 \mathrm{~mol} \%$ TBAB aqueous system in the presence of $0.028 \mathrm{~mol} \%$ DTAC vs. initial pressure at $277.15 \mathrm{~K}$.

mixture gas as the feed gas. As shown in Fig. 4, with the increase of the concentration of DTAC, the pressure drop $\left(\Delta \mathrm{P}_{1}\right)$ increases obviously. In the process of formation of gas hydrate, when the concentration of DTAC is less than CMC, the gas super-saturation in the solution increases, that is the amount of gas dissolving in water increases, with the increase of DTAC. When the concentration of DTAC is more than CMC, the DTAC molecules associate as the micelles containing solubilized gas, and the concentration of the micelles also increases with increasing concentration of DTAC resulting in the increase of the solubilized gas corresponding to the decrease of the pressure in the system and that is the increase of $\Delta \mathrm{P}_{1}$. Likewise, as shown in Fig. 4, the higher initial pressure causes the bigger pressure drop in the process of the hydrate formation. It attributes to the fact that more gas dissolves in the solution and forms the hydrate with the increase of the initial pressure, resulting in bigger pressure drop.

\section{3. $\mathrm{CO}_{2}$ concentration in hydrate slurry phase}

The effect of DTAC on promoting the gas to go into the hydrate slurry phase can be seen from Fig. 5, which shows the pressure increase $\left(\Delta \mathrm{P}_{2}\right)$ in the system after the hydrate dissociation vs. the initial pressure in the presence of DTAC with the different concentrations in $0.29 \mathrm{~mol} \% \mathrm{TBAB}$ aqueous solution at $274.95 \mathrm{~K}$ with $17.0 \mathrm{~mol} \% \mathrm{CO}_{2} / \mathrm{N}_{2}$ feed gas. As seen, the pressure increase $\left(\Delta \mathrm{P}_{2}\right)$ rises with the increases of the concentration of DTAC and initial pressure. It is noted that there is a remarkable increase of $\Delta \mathrm{P}_{2}$ due to the presence of DTAC with the concentration of $0.014 \mathrm{~mol} \%-0.056 \mathrm{~mol}$ $\%$, compared with that for the pure TBAB solution. It demonstrates that either DTAC or the increase of initial pressure can lead to the increase of the gas storage in the hydrate slurry phase.

The changes of $\mathrm{CO}_{2}$ concentration in the hydrate slurry vs. the initial pressures in the presence of DTAC with the different concentrations in $0.29 \mathrm{~mol} \%$ TBAB aqueous solution at $274.95 \mathrm{~K}$ with $17.0 \mathrm{~mol} \% \mathrm{CO}_{2} / \mathrm{N}_{2}$ feed gas are shown in Fig. 6 . As seen, the concentration of $\mathrm{CO}_{2}$ in hydrate slurry phase decreases with the increase of DTAC at the fixed initial pressure, and a substantial reduction occurs at the DTAC concentration of more than $0.028 \mathrm{~mol}$ $\%$. As mentioned above, the addition of DTAC lowers the surface tension of TBAB solution and promotes the dissolving ability of the mixture gas. Thus, it enhances not only the amount of $\mathrm{CO}_{2}$ but also the amount of $\mathrm{N}_{2}$ dissolved into the TBAB solution. However,

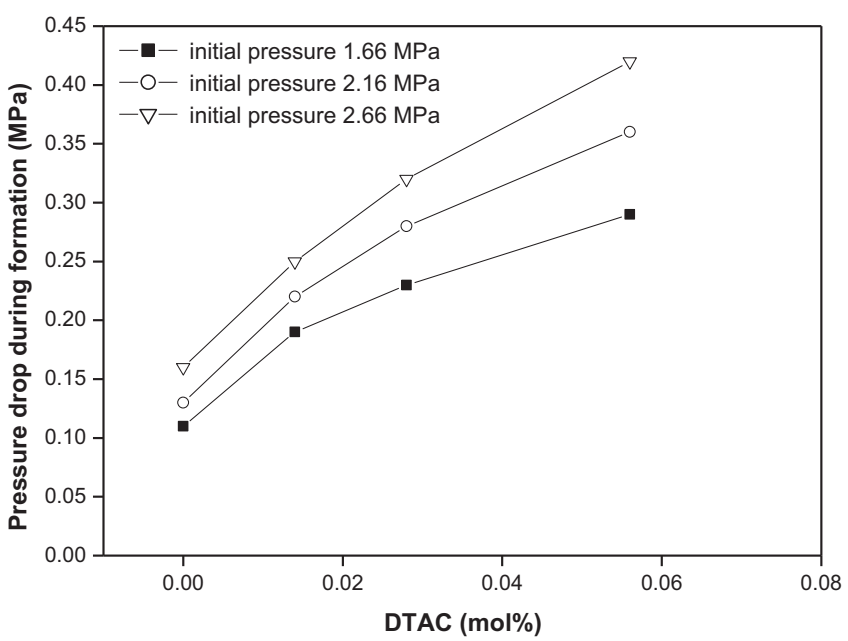

Fig. 4. Pressure drops $\left(\Delta \mathrm{P}_{1}\right)$ in the system vs. DTAC concentration in $0.29 \mathrm{~mol} \% \mathrm{TBAB}$ aqueous solution with different initial pressures at $274.95 \mathrm{~K}$ with $17.0 \mathrm{~mol} \% \mathrm{CO}_{2} / \mathrm{N}_{2}$ mixture gas.

relative to $\mathrm{CO}_{2}$, more $\mathrm{N}_{2}$ goes into the solution. This is due to the hydrophobic groups of DTAC molecules which preferentially adsorb $\mathrm{N}_{2}$ molecules and thus results in the reduction of $\mathrm{CO}_{2}$ concentration in the hydrate slurry phase with the increase of the DTAC concentration. In addition, when the concentration of DTAC is more than its CMC, the DTAC molecules in the solution form the Water/ Oil micelles, and, with the function of the hydrophobic group of the surfactant molecules micellized, substantial $\mathrm{N}_{2}$ can be preferentially enclosed into the micelles compared to $\mathrm{CO}_{2}$. This causes the remarkable reduction of concentration of $\mathrm{CO}_{2}$ at the DTAC concentration of $0.056 \mathrm{~mol} \%$, as shown in Fig. 6.

It is noted, as shown in Fig. 6 , that the $\mathrm{CO}_{2}$ concentration goes up with the increase of initial pressure, and then it goes down after an inflexion point occurs. The inflexion point is also the largest point of $\mathrm{CO}_{2}$ concentration. According to the phase equilibrium data given by Deschamps et al. [24] and Arjmandi et al. [25], $\mathrm{CO}_{2}$ is prior to form $\mathrm{CO}_{2}$ hydrate at low initial pressure, compared to $\mathrm{N}_{2}$. The driving force increases with the increase of initial pressure. Furthermore, $\mathrm{N}_{2}$ can compete with $\mathrm{CO}_{2}$ for hydrate cage $\left(5^{12}\right)$ occupancy with higher driving force [26] resulting in the dramatic

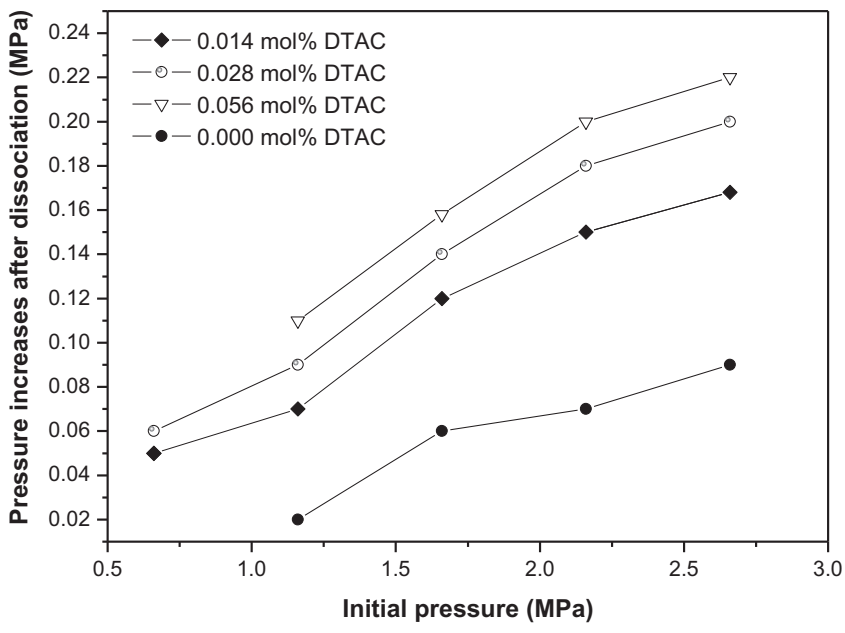

Fig. 5. Pressure increase $\left(\Delta \mathrm{P}_{2}\right)$ in the system after hydrate dissociation vs. initial pressure in the presence of DTAC with the different concentrations in $0.29 \mathrm{~mol} \% \mathrm{TBAB}$ aqueous solution at $274.95 \mathrm{~K}$ with $17.0 \mathrm{~mol} \% \mathrm{CO}_{2} / \mathrm{N}_{2}$ mixture gas. 


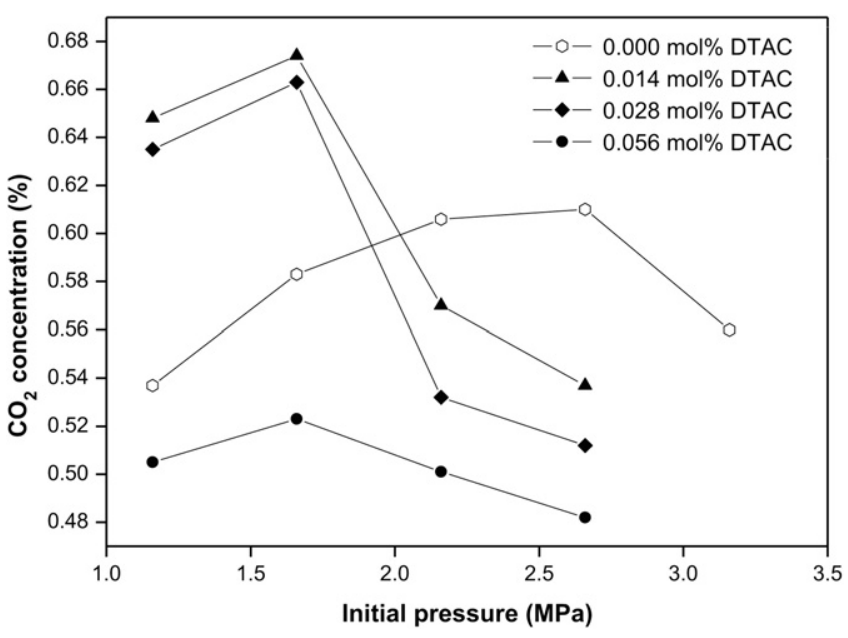

Fig. 6. $\mathrm{CO}_{2}$ concentration in hydrate slurry vs. initial pressures in the presence of DTAC with different concentration in $0.29 \mathrm{~mol} \% \mathrm{TBAB}$ aqueous solution at $274.95 \mathrm{~K}$ with $17.0 \mathrm{~mol} \% \mathrm{CO}_{2} / \mathrm{N}_{2}$ mixture gas.

decrease of the concentration of $\mathrm{CO}_{2}$ in the slurry phase after the inflexion point. The similar experimental phenomenon is also found by Fan et al. [27]. However, it can be found from Fig. 6 that the inflexion point for pure TBAB solution lags behind that for $\mathrm{TBAB}+\mathrm{DTAC}$ solution. Compared with those inflexion points occurring at the initial pressure of $1.66 \mathrm{MPa}$ in the presence of DTAC, the inflexion point in the absence of DTAC is shifted to 2.66 MPa. It may be attributed to the fact that the addition of DTAC actually lowers the equilibrium hydrate formation pressure of mixture gas. Meanwhile, the equilibrium pressures of $\mathrm{CO}_{2}$ and $\mathrm{N}_{2}$ are also lowered, resulting from the reduction of the surface tension with the function of the surfactant [28-32]. Thus, $\mathrm{N}_{2}$ can begin to compete with $\mathrm{CO}_{2}$ for hydrate cage $\left(5^{12}\right)$ occupancy at the relative lower driving force.

As discussed above, in order to shorten the induction time of hydrate and increase $\mathrm{CO}_{2}$ storage in the hydrate slurry phase to meet the requirement of $\mathrm{CO}_{2}$ separation from the flue gas ( $17.0 \mathrm{~mol}$ $\% \mathrm{CO}_{2} / \mathrm{N}_{2}$ mixture gas), it is found that the $0.29 \mathrm{~mol} \%$ $\mathrm{TBAB}+0.028 \mathrm{~mol} \% \mathrm{DTAC}$ aqueous solution is chosen as optimizing joint promoter for the $\mathrm{CO}_{2}$ separation at the initial pressure of

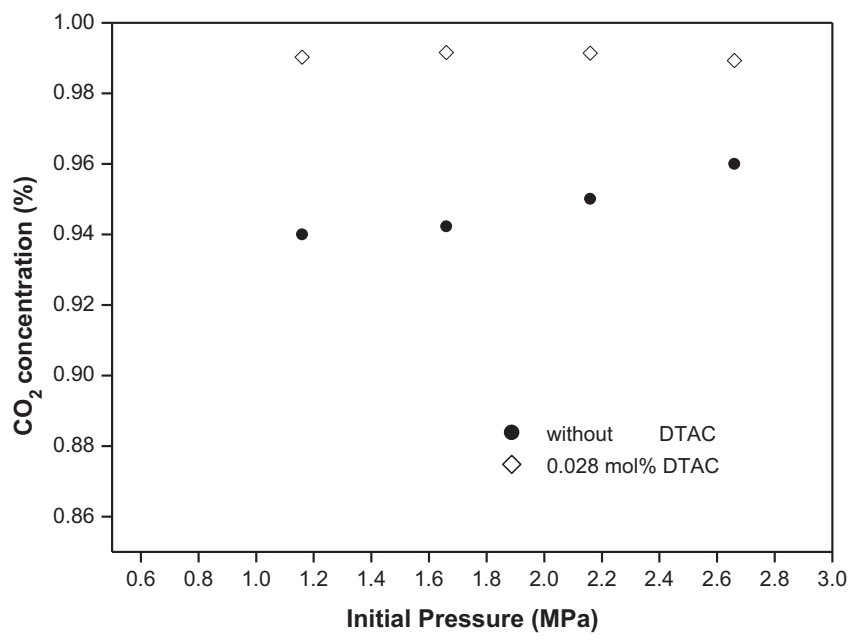

Fig. 7. $\mathrm{CO}_{2}$ concentration in hydrate slurry vs. initial pressures in the presence of $0.028 \mathrm{~mol} \%$ DTAC in $0.29 \mathrm{~mol} \%$ TBAB aqueous solution at $277.15 \mathrm{~K}$ with $65.0 \% \mathrm{CO}_{2} / \mathrm{N}_{2}$ mixture gas.

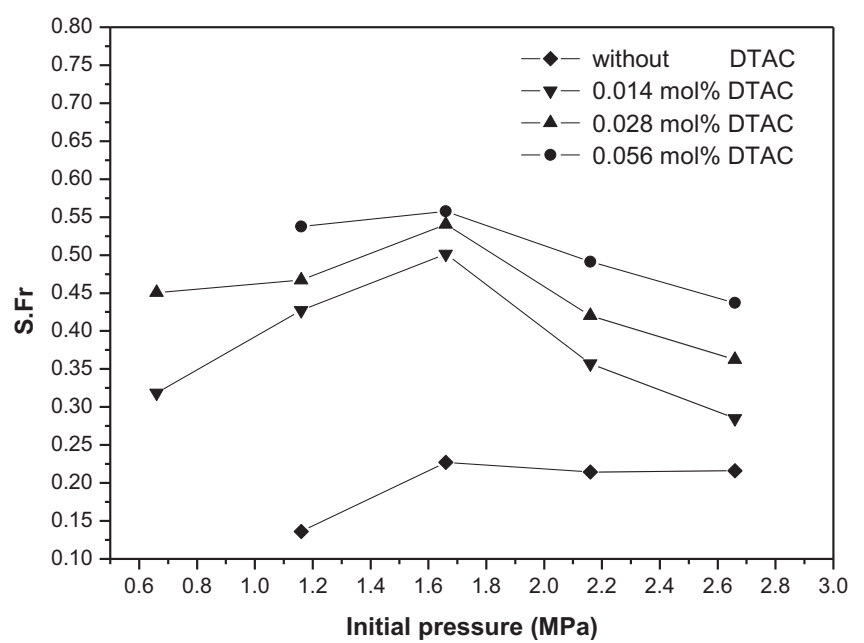

Fig. 8. Split fraction (S. Fr) vs. initial pressure in the presence of DTAC with different concentration in $0.29 \mathrm{~mol} \% \mathrm{TBAB}$ aqueous solution at $274.95 \mathrm{~K}$ with $17.0 \mathrm{~mol} \% \mathrm{CO}_{2} / \mathrm{N}_{2}$ mixture gas.

1.66 MPa. The similar result can also be obtained from the experiment in which the $65.0 \mathrm{~mol} \% \mathrm{CO}_{2} / \mathrm{N}_{2}$ gas mixture is used as the feed gas at $277.15 \mathrm{~K}$. As shown in Fig. 7, under the function of DTAC, the concentration of $\mathrm{CO}_{2}$ can be purified from $65.0 \mathrm{~mol} \%$ to $99.2 \mathrm{~mol} \%$ by forming the semi-clathrate hydrates at initial pressure $1.66 \mathrm{MPa}$ and temperature $277.15 \mathrm{~K}$. Compared to the operating pressure of $2.5 \mathrm{MPa}$ given by Linga et al.[6], the operating pressure to obtain $\mathrm{CO}_{2}$ of $99.2 \mathrm{~mol} \%$ in the stage 2 in this experiment is much lower. However, the highest concentration of $\mathrm{CO}_{2}$ in the hydrate slurry phase in the absence of DTAC is only $94.0 \mathrm{~mol} \%$ at $273.75 \mathrm{~K}$. Thereby, it is possible to realize the target of capturing $\mathrm{CO}_{2}$ through a two-stage hydrate separation in industry.

\subsection{Two-stage separation for $\mathrm{CO}_{2}$ recovery}

The separation efficiency with the two-stage hydrate separation was determined from the split fraction (S. Fr.) and the separation factor (S.F.) [9]. Fig. 8 shows the change of S. Fr. vs. the initial pressure in the presence of DTAC with the different concentrations in the aqueous solution of $0.29 \mathrm{~mol} \% \mathrm{TBAB}$ at $274.95 \mathrm{~K}$ with $17.0 \mathrm{~mol} \% \mathrm{CO}_{2} /$ $\mathrm{N}_{2}$ feed gas. As seen from Fig. 8, there is a significant enhancing of $S$. Fr.

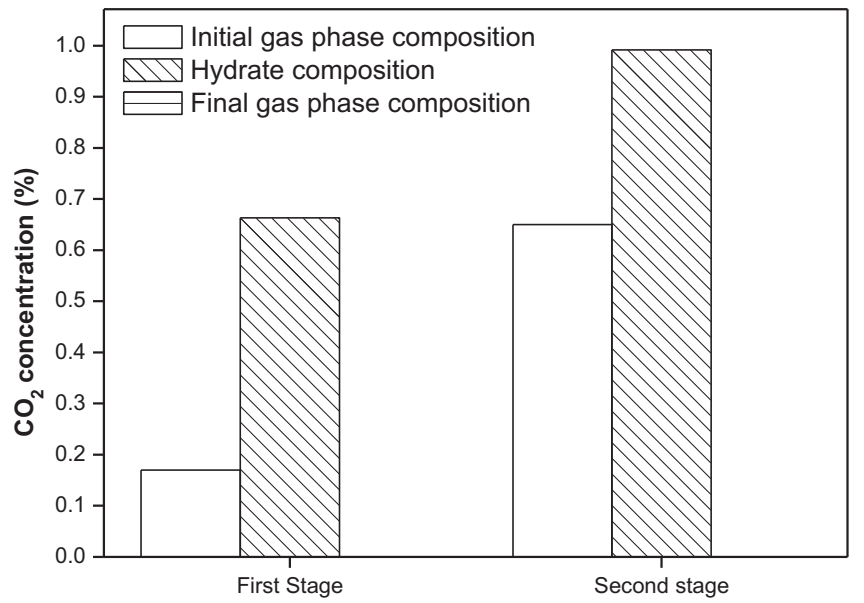

Fig. 9. $\mathrm{CO}_{2}$ concentration in feed gas, hydrate slurry phase and residual gas phase for two-stage separation with $0.028 \mathrm{~mol} \%$ DTAC in $0.29 \mathrm{~mol} \%$ TBAB aqueous solution at $1.66 \mathrm{MPa}$. 
Table 1

Split fraction and Separation factor for the two-stage $\mathrm{CO}_{2}$ separation.

\begin{tabular}{lllc}
\hline Feed gases & $\begin{array}{l}\text { Experimental } \\
\text { Conditions }\end{array}$ & $\begin{array}{l}\text { Split } \\
\text { Fraction }\end{array}$ & $\begin{array}{l}\text { Separation } \\
\text { Factor }\end{array}$ \\
\hline $17.0 \mathrm{~mol} \% \mathrm{CO}_{2} / \mathrm{N}_{2}$ & Stage 1 & 0.54 & 9.60 \\
& $274.95 \mathrm{~K}$ & & \\
& $1.66 \mathrm{MPa}$ & & \\
& DTAC $0.028 \mathrm{~mol} \%$ & & 62.25 \\
& Stage 2 & 0.39 & \\
& $277.15 \mathrm{~K}$ & & \\
& $1.66 \mathrm{MPa}$ & & \\
& DTAC $0.028 \mathrm{~mol} \%$ & & \\
&
\end{tabular}

in the presence of DTAC, compared with that without DTAC. The value of S. Fr. increases with the increase of the concentration of DTAC. However, the values of S. Fr. reach an extreme point with the four different DTAC concentrations of $0 \mathrm{~mol} \%, 0.014 \mathrm{~mol} \%, 0.028 \mathrm{~mol} \%$ and $0.056 \mathrm{~mol} \%$ when the initial pressure is $1.66 \mathrm{MPa}$, as shown in Fig. 8 , and the S. Fr. values with $0.028 \mathrm{~mol} \%$ and $0.056 \mathrm{~mol} \%$ DTAC are almost the same at the initial pressure of $1.66 \mathrm{MPa}$. Hence, the operational condition at the DTAC concentration of $0.028 \mathrm{~mol} \%$ and the initial pressure of $1.66 \mathrm{MPa}$ is an optimal one for the $\mathrm{CO}_{2}$ separation via the hydration crystallization.

Fig. 9 gives $\mathrm{CO}_{2}$ concentration in the feed gas, the hydrate slurry phase and the residual gas phase for two-stage separation with $0.028 \mathrm{~mol} \% \mathrm{DTAC}$ at $1.66 \mathrm{MPa}$. The results indicate that after the separation for the $\mathrm{CO}_{2} / \mathrm{N}_{2}$ mixture gas (simulated flue gas) at $274.95 \mathrm{~K}$ and $1.66 \mathrm{MPa}$, a $\mathrm{CO}_{2}$-rich gas containing more than $65.0 \mathrm{~mol} \% \mathrm{CO}_{2}$ can be obtained, as shown in Figs. 6 and 9. As the industrial objective is to obtain purified $\mathrm{CO}_{2}$ with the high concentration, $65.0 \mathrm{~mol} \% \mathrm{CO}_{2}$ gas needs to be treated further with a second-stage hydrate separation. As seen in Figs. 7 and 9, the $\mathrm{CO}_{2}$ content is increased from initial $65.0 \mathrm{~mol} \%$ to $99.2 \%$ after the second separation stage at $277.15 \mathrm{~K}$ and $1.66 \mathrm{MPa}$.

Table 1 shows the values of the split fraction and the separation factor for the two-stage $\mathrm{CO}_{2}$ separation with the DTAC concentration of $0.028 \mathrm{~mol} \%$ at the initial pressure of $1.66 \mathrm{MPa}$. It can be seen from Table 1 that $\mathrm{CO}_{2}$ split fractions for Stage 1 and Stage 2 are 0.54 and 0.39 , respectively, and the separation factors are 9.60 and 62.25 , respectively, which are superior to those given by Linga et al. [6].

\section{Conclusion}

The induction time of the hydrate formation and $\mathrm{CO}_{2}$ recovery from the flue gas $\left(\mathrm{N}_{2} / \mathrm{CO}_{2}\right)$ mixture are investigated in $0.29 \mathrm{~mol} \%$ TBAB aqueous solution with the functions of DTAC and the different initial pressures. The result shows that DTAC as a surfactant considerably shortens the induction time of hydrate formation. The induction time decreases dramatically with the increase of the concentration of DTAC. However, when the concentration exceeds its CMC value, the DTAC micelles forms and the induction time cannot be shortened any more. Meanwhile, substantial $\mathrm{N}_{2}$ is preferentially solubilized into the micelles compared to $\mathrm{CO}_{2}$, resulting in the remarkable reduction of $\mathrm{CO}_{2}$ recovery. Therefore, the optimizing concentration of DTAC for the $\mathrm{CO}_{2}$ separation is its $\mathrm{CMC}$, approximately $0.028 \mathrm{~mol} \%$. On the other hand, the induction time reduces and the $\mathrm{CO}_{2}$ concentration in the hydrate slurry phase increases with the increase of initial pressure. However, when the pressure is more than $1.66 \mathrm{MPa}$, the $\mathrm{CO}_{2}$ concentration in the hydrate slurry phase and $\mathrm{CO}_{2}$ separation efficiency reduce due to the increase of the concentration of $\mathrm{N}_{2}$ which can compete with $\mathrm{CO}_{2}$ for hydrate cage $\left(5^{12}\right)$ occupancy. Hence, the initial pressure of $1.66 \mathrm{MPa}$ in conjunction with 0.028 mol\% DTAC is most favorable for $\mathrm{CO}_{2}$ separation. At this condition, the induction time can be shortened to $4.0 \mathrm{~min}$ for the first stage separation and $0.4 \mathrm{~min}$ for second-stage separation These are only one eighth and one thirteenth of those without DTAC. Meanwhile, $\mathrm{CO}_{2}$ can be purified from $17.0 \mathrm{~mol} \%$ to $99.2 \mathrm{~mol} \%$ with the two-stage hydrate separation process, $\mathrm{CO}_{2}$ split fractions for Stage 1 and Stage 2 are 0.54 and 0.39, respectively, and the separation factors are 9.60 and 62.25 , respectively.

\section{Acknowledgments}

This work was supported by National Natural Science Foundation of China (20676133, 20773133), Science \& Technology Program of Guangdong Province (2009B050600006),CAS Magnitude Science and Technology Apparatus Development Program (YZ200717), and CAS Knowledge Innovation Program (KGCX2-YW3X6), which are gratefully acknowledged.

\section{References}

[1] Happel J, Hnatow MA, Meyer H. The study of separation of nitrogen from methane by hydrate formation using a novel apparatus. International Conference on Natural Gas Hydrates 1994;715:412-424.

[2] Duc NH, Chauvy F, Herri JM. $\mathrm{CO}_{2}$ capture by hydrate crystallization - A potential solution for gas emission of steelmaking industry. Energy Conversion and Management 2007;48(4):1313-22.

[3] Glew DN, Rath NS. Variable composition of chlorine and ethylene oxide clathrate hydrates. Journal of Chemical Physics 1966;44(4):1710-1.

[4] Happel J. New vistas for gas hydrate research and technology. International Conference on Natural Gas Hydrates 1994;715:564-569.

[5] Spencer BF. Civil engineering applications of smart damping technology. In: Proceedings of the 5th International Conference on Vibration Engineering. 2002;771-782.

[6] Linga P, Adeyemo A, Englezos P. Medium-pressure clathrate hydrate/ membrane hybrid process for postcombustion capture of carbon dioxide. Environmental Science \& Technology 2008;42(1):315-20.

[7] Kang SP, Lee H, Lee CS, Sung WM. Hydrate phase equilibria of the guest mixtures containing $\mathrm{CO}_{2}, \mathrm{~N}_{2}$ and tetrahydrofuran. Fluid Phase Equilibria 2001;185(1-2):101-9.

[8] Kang SP, Lee $\mathrm{H}$. Recovery of $\mathrm{CO}_{2}$ from flue gas using gas hydrate: thermodynamic verification through phase equilibrium measurements. Environmental Science \& Technology 2000;34(20):4397-400.

[9] Linga P, Kumar R, Englezos P. The clathrate hydrate process for post and precombustion capture of carbon dioxide. Journal of Hazardous Materials 2007;149(3):625-9.

[10] Ding T. Gas hydrate to capture and sequester $\mathrm{CO}_{2}$. Mississippi: Mississippi State University; 2004.

[11] Li SF, Fan SS, Wang JQ Lang XM, Liang DQ. $\mathrm{CO}_{2}$ capture from binary mixture via forming hydrate with the help of tetra- $n$-butyl ammonium bromide. Journal of Natural Gas Chemistry 2009;18(1):15-20.

[12] Lipkowski J, Komarov VY, Rodionova TV, Aladko LS. X-ray investigation of compounds crystallized in aqueous solution of tetrabutylammonium laurate. The structure of $\left(\mathrm{C}_{4} \mathrm{H}_{9}\right)_{4} \mathrm{~N}\left(\mathrm{C}_{11} \mathrm{H}_{23} \mathrm{COO}\right) \cdot 3 \mathrm{C}_{11} \mathrm{H}_{23} \mathrm{COOH} \cdot 4 \mathrm{H}_{2} \mathrm{O}$. Journal of Structural Chemistry 2005;46:S51-7.

[13] Oyama H, Shimada W, Ebinuma T, Kamata Y, Takeya S, Uchida T, et al. Phase diagram, latent heat, and specific heat of TBAB semi-clathrate hydrate crystals. Fluid Phase Equilibria 2005;234(1-2):131-5.

[14] Darbouret M, Cournil M, Herri JM. Rheological study of TBAB hydrate slurries as secondary two-phase refrigerants. International Journal of RefrigerationRevue Internationale Du Froid 2005;28(5):663-71.

[15] Farid A. Gas separation and storage using semi-clathrate hydrates. 2008.

[16] Oyama $H$. Phase behavior of TBAB semi-clathrate hydrate crystal under several vapor components. 2008.

[17] Zhang Tao LY, Li Xiaosen, Chen Zhaoyang, Yan Kefeng. The study on hydrate equilibrium formation conditions of $\mathrm{CO}_{2} \mathrm{~N}_{2} \mathrm{TBAB}$ and $\mathrm{CO}_{2} \mathrm{~N}_{2} \mathrm{THF}$ system. Chinese Journal of Process Engineering; 2009. 2009.9.

[18] Fang KJ, Jiang X, Wang CX, Wu M, Yan Y. Properties of the nanoscale hydrophilic cationic pigment based on quaternary surfactant. Journal of Dispersion Science and Technology 2008;29(1):52-7.

[19] Natarajan V, Bishnoi PR, Kalogerakis N. Induction phenomena in gas hydrate nucleation. Chemical Engineering Science 1994;49(13):2075-87.

[20] Clint J. Surfactant aggregation. New York: Chapman and Hall; 1992.

[21] Myers D. Surfactant science and technology. 2nd ed. 1992.

[22] Zhong Y, Rogers RE. Surfactant effects on gas hydrate formation. Chemical Engineering Science 2000;55(19):4175-87.

[23] Watanabe K, Imai S, Mori YH. Surfactant effects on hydrate formation in an unstirred gas/liquid system: an experimental study using HFC-32 and sodium dodecyl sulfate. Chemical Engineering Science 2005;60(17):4846-57.

[24] Deschamps J, Dalmazzone D. Dissociation enthalpies and phase equilibrium for TBAB semi-clathrate hydrates of $\mathrm{N}_{2}, \mathrm{CO}_{2}, \mathrm{~N}_{2}+\mathrm{CO}_{2}$ and $\mathrm{CH}_{4}+\mathrm{CO}_{2}$. Journal of Thermal Analysis and Calorimetry 2009;98(1):113-8.

[25] Arjmandi M, Chapoy A, Tohidi B. Equilibrium data of hydrogen, methane, nitrogen, carbon dioxide, and natural gas in semi-clathrate hydrates of 
tetrabutyl ammonium bromide. Journal of Chemical and Engineering Data 2007;52(6):2153-8.

[26] Seo YT, Lee H. Structure and guest distribution of the mixed carbon dioxide and nitrogen hydrates as revealed by X-ray diffraction and ${ }^{13} \mathrm{C}$ NMR spectroscopy. Journal of Physical Chemistry B 2004;108(2):530-4.

[27] Fan SS, Li SF, Wang JQ Lang XM, Wang YH. Efficient capture of $\mathrm{CO}_{2}$ from simulated flue gas by formation of TBAB or TBAF semi-clathrate hydrates. Energy \& Fuels 2009;23(8):4202-8.

[28] Daimaru T, Yamasaki A, Yanagisawa Y. Effect of surfactant carbon chain length on hydrate formation kinetics. Journal of Petroleum Science and Engineering 2007;56(1-3):89-96.
[29] Kalogerakis N, Jamaluddin AKM, Dholabhai PD, Bishnoi PR. Effect of surfactants on hydrate formation kinetics. Society of Petroleum Engineers; 1993. 10.2118/25188-MS:25188-MS

[30] Karaaslan U, Parlaktuna M. Promotion effect of polymers and surfactants on hydrate formation rate. Energy \& Fuels 2002;16(6):1413-6.

[31] Okutani K, Kuwabara Y, Mori YH. Surfactant effects on hydrate formation in an unstirred gas/liquid system: an experimental study using methane and sodium alkyl sulfates. Chemical Engineering Science 2008;63(1):183-94.

[32] Rogers R, Zhang G, Dearman J, Woods C. Investigations into surfactant/gas hydrate relationship. Journal of Petroleum Science and Engineering 2007;56 $(1-3): 82-8$. 